\title{
Gravitational production of dark matter in the Peebles-Vilenkin model
}

\author{
Jaume Haro $^{\mathrm{a}}$ \\ Departament de Matemàtiques, Universitat Politècnica de Catalunya, Diagonal 647, 08028 Barcelona, Spain
}

Received: 18 April 2019 / Accepted: 2 March 2020 / Published online: 19 March 2020

(C) The Author(s) 2020

\begin{abstract}
The gravitational production of superheavy dark matter, in the Peebles-Vilenkin quintessential inflation model, is studied in two different scenarios: When the particles, whose decay products reheat the universe after the end of the inflationary period, are created gravitationally, and when are produced via instant preheating. We show that the viability of both scenarios requires that the mass of the superheavy dark matter to be approximately between $10^{16}$ and $10^{17} \mathrm{GeV}$.
\end{abstract}

\section{Introduction}

Quintessential inflation, which was addressed for the first time by Peebles and Vilenkin (PV) in [1], is an attempt to unify inflation and quintessence via a single scalar field whose potential allows inflation while at late time provides quintessence (see for instance [2]). A remarkable property of the PV model is that it contains an abrupt phase transition from inflation to kination (a regime where all the energy density of the inflation turns into kinetic), where the adiabatic regime is broken and, thus, particles could be gravitationally created $[3,4]$. This leads to the possibility to explain the abundance of dark matter through the gravitational production of superheavy particles [5,6], although gravitational production of dark matter could also occur in standard inflation during the oscillations of the inflaton field [7-9] (see also the early papers [10-12]).

Considering the gravitational production of two kinds of superheavy particles: $X$-particles, conformally coupled with gravity, whose energy density after their decay and later thermalization of decay products will dominate the energy density of the scalar field in order to match with the Hot Big Bang (HBB), and dark $Y$-particles which are only gravitationally interacting massive particles (GIMP), we will show that the PV model preserves the Big Bang Nucleosynthesis (BBN) success. More precisely, the over-

a e-mail: jaime.haro@upc.edu (corresponding author) production of Gravitational Waves (GWs) does not disturb the BBN for $X$-particles and $Y$-particles with masses in the range of $10^{15}-10^{16} \mathrm{GeV}$ and $10^{16}-10^{17} \mathrm{GeV}$ respectively, leading to a maximum reheating temperature in the $\mathrm{GeV}$ regime.

On the contrary, for massless conformally coupled $X$ particles produced via instant preheating (see [13] for a detailed discussion of this mechanism of particle creation), assuming that $Y$-GIMP, which are gravitationally produced, are the constituent of the dark matter, the viability of the model requires that the mass of the superheavy $Y$-particles is approximately of the order of $10^{16} \mathrm{GeV}$, yielding a reheating temperature around $10^{8} \mathrm{GeV}$.

The work is structured as follows: In Sect. 2 and improved version of the well-known Peebles-Vilenkin model for quintessential inflation is presented. Section 3 is devoted to the study of gravitational production of superheavy $X$ particles whose decaying products reheat the universe and superheavy $Y$-particles which are the responsible for the abundance of dark matter. In addition, we show how to overpass the constrains coming from the overproduction of gravitational waves. In Sect. 4 we consider the case in which the $X$-particles are produced via instant preheating. The dynamics of the scalar field, for the improved model proposed in Sect. 2, is studied in detail in Sect. 5, and finally, we present the conclusions of our study in Sect. 6 .

\section{The Peebles-Vilenkin model}

It is well-known that in quintessential inflation the number of e-folds from the pivot scale exiting the Hubble radius to the end of inflation is greater than 60. For this reason, in order that the theoretical values of the spectral index and the ratio of tensor to scalar perturbations enters in their marginalized joint confidence contour in the plane $\left(n_{s}, r\right)$ at $2 \sigma$ C.L., we have changed the quartic inflationary potential of the origi- 


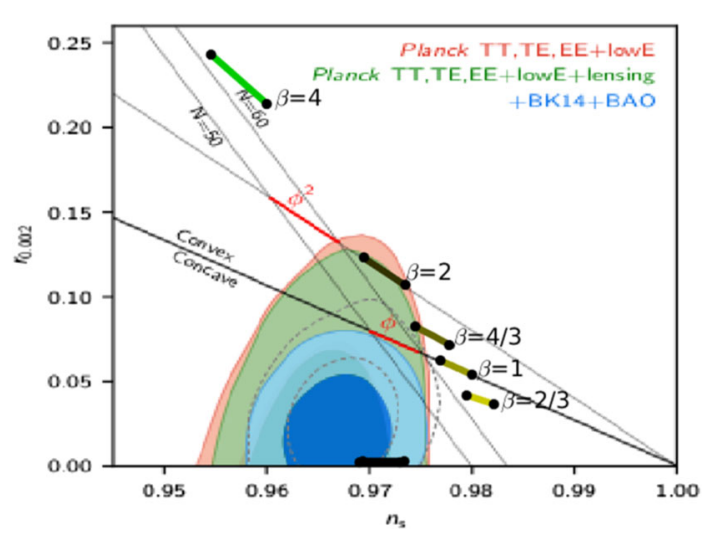

Fig. 1 Marginalized joint confidence contours for $\left(n_{s}, r\right)$ at $1 \sigma$ and $2 \sigma$ confidence level (CL). Considering the inflationary piece of the potential as $V=\lambda \phi^{\beta}$, in quintessential inflation, for the values of $\beta=4,2,3 / 4,1,2 / 3$, we have drawn the curves from 65 to 75 e-folds (see the green, which correspond to the original P-V model, and black curves). And when one considers the standard inflation, for $\beta=2,1$, the curves have been drawn in red from 50 to $60 e$-folds. As one can see, the quadratic potential $\left(V \propto \phi^{2}\right)$, which is disregarded in standard inflation at greater than $2 \sigma$ CL from a combination of Planck and BICEP2 limits on the tensor-to-scalar ratio [15], is favored for some likelihoods in quintessential inflation. In the lower part of the image there is the curve for the potential (2). The value of $r$ is nearly 0 and, if considering all Planck likelihoods, it stands within the $2 \sigma$ CL for $65 \lesssim N \lesssim 75$

nal PV quintessential inflation model with a quadratic one, obtaining:

$V(\varphi)=\left\{\begin{array}{cl}\frac{1}{2} m^{2}\left(\varphi^{2}+M^{2}\right) & \text { for } \quad \varphi \leq 0 \\ \frac{1}{2} m^{2} \frac{M^{6}}{\varphi^{4}+M^{4}} & \text { for } \varphi \geq 0,\end{array}\right.$

where $m$ is the mass of the scalar field and $M \sim 10 \mathrm{GeV}$, is an small mass that has to be calculated numerically [14].

As we can see in the Fig. 1, the spectral index and the tensor/scalar ratio enter perfectly in the two dimensional marginalized joint confidence contour at $2 \sigma$ Confidence Level (CL) for the Planck TT, TE, EE + low E and for the Planck TT, TE, EE + low E + lensing likelihood [15]. In addition, if one wants that the model enters, at $2 \sigma \mathrm{CL}$, in the contour for the Planck TT, TE, EE + low E + lensing + BK14+BAO likelihood, i.e., taking into account gravitational waves, one has to replace the inflationary piece of the potential by a plateau-like potential [16] or $\alpha$-attractors [1719] such as an Starobinsky-type potential [20]

$V(\varphi)=\left\{\begin{array}{cl}\lambda M_{p l}^{4}\left(1-e^{\sqrt{\frac{2}{3}} \frac{\varphi}{M_{p l}}}\right)^{2}+\lambda \tilde{M}^{4} & \text { for } \varphi \leq 0 \\ \lambda \frac{\tilde{M}^{8}}{\varphi^{4}+\tilde{M}^{4}} & \text { for } \varphi \geq 0,\end{array}\right.$

where $\lambda$ is a dimensionless parameter of the order $10^{-10}$, and now $\tilde{M} \sim 10^{5} \mathrm{GeV}$ (see [1]). Effectively, for the potential (2) one has (see for instance [19]) $n_{s} \cong 1-\frac{2}{N}, \quad$ and $\quad r \cong \frac{12}{N^{2}}$,

where $N$ is the number of e-folds. Thus, as we have already explained, since in quintessential inflation the number of efolds is greater than 60 one gets that $r<0.0034$, and clearly, the spectral index and the tensor/scalar ratio enters at $2 \sigma \mathrm{CL}$, in the contour for the Planck TT, TE, EE + low E + lensing +BK14+BAO likelihood (see Fig. 1).

Remark 2.1 The first piece of the potential (2) is obtained when one deals with $R^{2}$ gravity in the Einstein Frame [20], and the tail, which is the same used in [1], comes from SUSY QED [21].

Remark 2.2 The second derivative of the potentials (1) and (2) has a jump discontinuity at the beginning of kination, but its physical origin is not discussed in the present work. However, one may argue, as was shown in [22] where the discontinuity of the second derivative of the potential appears during inflation, that its origin could be due to a second-order phase transition of another scalar field coupled with the field $\varphi$. This is a point that deserves future investigation.

To calculate $H_{k i n}$, the value of the Hubble parameter at the beginning of kination for the model (2), first of all we calculate the slow roll parameters: Denoting by $\epsilon_{*}=$ $\frac{M_{p l}^{2}}{2}\left(\frac{V_{\varphi}\left(\varphi_{*}\right)}{V\left(\varphi_{*}\right)}\right)^{2}$ and $\eta_{*}=M_{p l}^{2} \frac{V_{\varphi \varphi}\left(\varphi_{*}\right)}{V\left(\varphi_{*}\right)}$ the values of the slow roll parameters and by $\varphi_{*}$ the value of the scalar field when the pivot scale exits the Hubble radius, since the mass $\tilde{M}$ satisfies $\tilde{M} \ll M_{p l}$, one has $\epsilon_{*} \cong \frac{4}{3} e^{2 \sqrt{\frac{2}{3}} \frac{\varphi_{*}}{M_{p l}}} \eta_{*}=-\frac{4}{3} e^{\sqrt{\frac{2}{3} \frac{\varphi_{*}}{M_{p l}}}}$, and thus, the spectral index is given by [23]

$1-n_{s} \cong 6 \epsilon_{*}-2 \eta_{*} \cong \frac{8}{3} e^{\sqrt{\frac{2}{3} \frac{\varphi_{*}}{M_{p l}}}}$,

meaning that

$\varphi_{*} \cong \sqrt{\frac{3}{2}} M_{p l} \ln \left(\frac{3}{8}\left(1-n_{s}\right)\right)$.

On the other hand, the observational estimation of the power spectrum of the scalar perturbations when the pivot scale leaves the Hubble radius is $\mathcal{P}_{\zeta} \cong \frac{H_{*}^{2}}{8 \pi^{2} M_{p l}^{2} \epsilon_{*}} \sim 2 \times 10^{-9}$ [23]. Since during the slow roll regime the kinetic energy density is negligible compared with the potential one, we will have $H_{*}^{2} \cong \frac{\lambda}{3} M_{p l}^{2}$, and using the relation $\epsilon_{*}=\frac{3}{16}\left(1-n_{s}\right)^{2}$ one gets

$\lambda \sim 9 \pi^{2}\left(1-n_{s}\right)^{2} \times 10^{-9}$.

Taking into account that the observational value of the spectral index is $n_{s}=0.968 \pm 0.006$ [24], if one chooses its central value one gets

$\lambda=9 \times 10^{-11}$ and $\varphi_{*} \cong-5.42 M_{p l}$. 


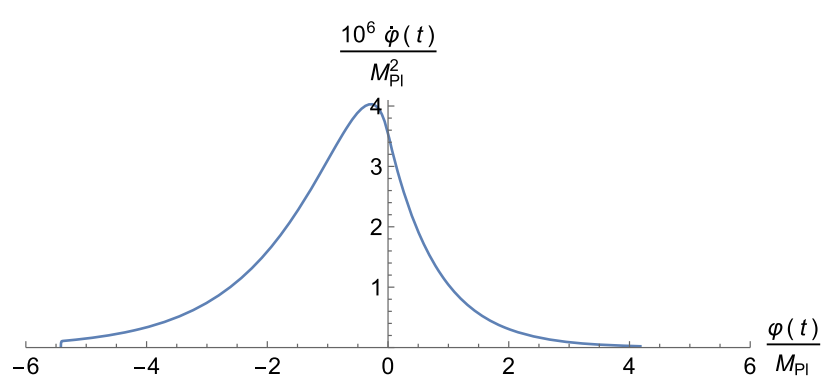

Fig. 2 Evolution of the velocity of the scalar field obtained integrating the Eq. (8) with initial conditions when the pivot scale leaves the Hubble horizon, i.e., for $\varphi_{*}=-5.42 M_{p l}$ and $\dot{\varphi}_{*}=0$

Now, taking into account that inflation ends when $\epsilon=1$, that is, when $e^{\sqrt{\frac{2}{3} \frac{\varphi}{M_{p l}}}}=\sqrt{3}(2-\sqrt{3})$. This means that the value of the potential energy at the end of inflation is approximately $\frac{3}{2} \lambda(4-2 \sqrt{3})^{2} M_{p l}^{4}$ which is many orders greater than $V(0)=\lambda \tilde{M}^{4}$, because $\tilde{M} \ll M_{p l}$. Thus, we can safely conclude that the kination phase, i.e. when practically all energy density is kinetic, has already started when the field $\varphi$ crosses the origin (see also the Fig. 2, where one can see that the maximum of the velocity of the scalar field is obtained very close to $\varphi=0$.). Then, to simplify, we can consider that kination starts at $\varphi_{\text {kin }}=0$, and to obtain the value of the Hubble rate at the beginning of kination, namely $\mathrm{H}_{k i n}$, we have to solve numerically the conservation equation

$\ddot{\varphi}+3 \sqrt{\frac{\frac{\dot{\varphi}^{2}}{2}+V(\varphi)}{3 M_{p l}^{2}}} \dot{\varphi}+V_{\varphi}=0$,

with initial conditions $\varphi_{*}=-5.42 M_{p l}$ and $\dot{\varphi}_{*}=0$ (obviously, one can choose other similar initial conditions and the result has to be practically the same, because the inflationary dynamics is an attractor).

Using event-driven integration with an ode RK78 integrator, when $\varphi$ vanishes one gets $\dot{\varphi}_{k i n}=3.54 \times 10^{-6} M_{p l}^{2}$, and thus

$H_{k i n}=\frac{\dot{\varphi}_{k i n}}{\sqrt{6} M_{p l}} \cong 1.44 \times 10^{-6} M_{p l}$,

and

$\rho_{\varphi, k i n} \cong 6.26 \times 10^{-12} M_{p l}^{4}$.

Coming back to the PV model (1), one has $\epsilon_{*}=\eta_{*}=$ $\frac{2 M_{p l}^{2}}{\varphi_{*}^{2}}$, and thus, the spectral index is given by

$$
\begin{gathered}
1-n_{s}=6 \epsilon_{*}-2 \eta_{*}=\frac{8 M_{p l}^{2}}{\varphi_{*}^{2}} \\
\Longrightarrow \varphi_{*}=-\sqrt{\frac{8}{1-n_{s}}} M_{p l},
\end{gathered}
$$

and using the formula of the power spectrum of scalar perturbations one gets

$m \sim \sqrt{\frac{3}{10}} \pi\left(1-n_{s}\right) \times 10^{-4} M_{p l}$,

which for $n_{s}=0.968$, leads to

$m=5 \times 10^{-6} M_{p l}$ and $\varphi_{*}=-15.81 M_{p l}$.

Once again, using event-driven integration with an ode RK78 integrator one gets $\dot{\varphi}_{k i n}=2.34 \times 10^{-6} M_{p l}^{2}$, and thus

$H_{k i n}=\frac{\dot{\varphi}_{k i n}}{\sqrt{6} M_{p l}} \cong 9.5 \times 10^{-7} M_{p l}$,

and

$\rho_{\varphi, k i n} \cong 2.73 \times 10^{-12} M_{p l}^{4}$.

To end this Section, note that, for the model (2), at the beginning of kination the energy density of the inflation is $\rho_{\varphi, k i n} \cong 6.26 \times 10^{-12} M_{p l}^{4}$, which shows that the energy density drops an order of magnitude between the end of inflation and the beginning of kination, because at the end of inflation the effective Equation of State (EoS) parameter $w_{\text {eff }}=\frac{P}{\rho}$ is equal to $-1 / 3$, meaning that, at that moment, $\dot{\varphi}^{2}=V(\varphi)$, i.e. $\rho=\frac{3}{2} V$, and thus, at the end of inflation, as we have already seen, when $\epsilon=1 \Longrightarrow e^{\sqrt{\frac{2}{3} \frac{\varphi}{M_{p l}}}}=\sqrt{3}(2-\sqrt{3})$ the energy density is given by $\frac{3}{2} \lambda(4-2 \sqrt{3})^{2} M_{p l}^{4} \cong 3.8 \times 10^{-11} M_{p l}^{4}$. Finally, note that the same happens for the model (1).

\section{Reheating via gravitational particle production}

Since the second derivative of the potential (2) is discontinuous at $\varphi=0$, from the conservation equation one can see that the third temporal derivative of the inflation field is discontinuous at the beginning of kination, and using the Raychaudhuri equation $\dot{H}=-\frac{\dot{\varphi}^{2}}{2 M_{p l}^{2}}$ one can deduce that at the beginning of kination the third derivative of the Hubble parameter is discontinuous, enhancing the particle production as discussed in [25].

Then, in order that vacuum polarization effects do not disturb the dynamics of the $\varphi$-field, the mass of superheavy $A$-particles, produced gravitationally, has to satisfy $m_{A} \gg H_{B} \gg m$, where we have assumed that the beginning of inflation occurs at GUT scales, that is, when the Hubble parameter is of the order $H_{B} \sim 10^{14} \mathrm{GeV}$ (see for instance [26]). For this reason, the mass of superheavy particles must satisfy $m_{A} \geq 10^{15} \mathrm{GeV}$.

In fact, in the conformally coupled case, the $k$-mode satisfy the equation

$\chi_{k}^{\prime \prime}+\omega_{k}^{2}(\tau) \chi_{k}=0$, 
where the derivative is with respect the conformal time and $\omega_{k}(\tau)=\sqrt{k^{2}+a^{2}(\tau) m_{A}^{2}}$ is the time dependent frequency.

Note that the jump discontinuity of the third derivative of the Hubble rate is equivalent to a jump discontinuity of the fourth derivative of the frequency $\omega_{k}(\tau)$, and thus its fifth temporal derivative is like a Dirac's delta, so for a smoother version of the potential (2) the discontinuity of the second derivative of the potential could be replaced by the no-adiabatic condition $\frac{1}{\omega_{k}^{6}(\tau)} \frac{d^{5} \omega(\tau)}{d \tau^{5}} \geq 1$ during a short period of time centered at the beginning of kination. However, a smoother potential hinders the possibility to obtain analytic expressions of the energy density of the produced particles, and for this reason we will continue with the potential (2).

Then, using the WKB approximation up to order two

$\chi_{2, k}^{W K B}(\tau) \equiv \sqrt{\frac{1}{2 W_{2, k}(\tau)}} e^{-i \int^{\tau} W_{2, k}(s) d s}$,

where $W_{2, k}$ has the following complicated form [27]

$$
\begin{aligned}
W_{2, k}= & \omega_{k}-\frac{m^{2} a^{4}}{4 \omega_{k}^{3}}\left(\dot{H}+3 H^{2}\right)+\frac{5 m^{2} a^{6}}{8 \omega_{k}^{5}} H^{2} \\
& +\frac{m^{2} a^{6}}{16 \omega_{k}^{5}}\left(\dddot{H}+15 \ddot{H} H+10 \dot{H}^{2}+86 \dot{H} H^{2}+60 H^{4}\right) \\
& -\frac{m^{4} a^{8}}{32 \omega_{k}^{7}}\left(28 \ddot{H} H+19 \dot{H}^{2}+394 \dot{H} H^{2}+507 H^{4}\right) \\
& +\frac{221 m^{6} a^{10}}{32 \omega_{k}^{9}}\left(\dot{H}+3 H^{2}\right) H^{2}-\frac{1105 m^{8} a^{12}}{128 \omega_{k}^{11}} H^{4},
\end{aligned}
$$

one can find the Bogoliubov coefficients of the $k$-mode, namely $\alpha_{k}$ and $\beta_{k}$, matching the mode (17) with the combination $\alpha_{k} \chi_{2, k}^{W K B}(\tau)+\beta_{k}\left(\chi_{2, k}^{W K B}\right)^{*}(\tau)$ at $\varphi_{k i n}=0$, i.e., when the third derivative of the Hubble rate is discontinuous. Denoting by $\tau_{k i n}$ this time, we will have $\beta_{k}=$ $i \mathcal{W}\left[\chi_{2, k}^{W K B}\left(\tau_{\text {kin }}^{-}\right), \chi_{2, k}^{W K B}\left(\tau_{\text {kin }}^{+}\right)\right]$, where $\mathcal{W}$ is the Wronskian, and we have used the notation $f\left(\tau_{\text {kin }}^{ \pm}\right)=\lim _{\tau \rightarrow \tau_{\text {kin }}^{ \pm}} f(\tau)$, i.e., $f\left(\tau_{\text {kin }}^{ \pm}\right)$denotes the limit on the right and on the left of the point $\tau_{k i n}$.

Then, the leading term of the $\beta_{k}$-Bogoliubov coefficient is $\frac{1}{2}\left(\frac{W_{2, k}\left(\tau_{\text {kin }}^{-}\right)-W_{2, k}\left(\tau_{\text {kin }}^{+}\right)}{\sqrt{W_{2, k}\left(\tau_{\text {kin }}^{+}\right) W_{2, k}\left(\tau_{\text {kin }}^{-}\right)}}\right)$, and thus, form the expression (18), one can see that the discontinuous term is $\frac{m^{2} a^{6}}{16 \omega_{k}^{5}} \dddot{H}$, meaning that

$\left|\beta_{k}\right|^{2} \cong \frac{m_{A}^{4} a_{k i n}^{12}\left(\dddot{H}\left(\tau_{k i n}^{-}\right)-\dddot{H}\left(\tau_{k i n}^{+}\right)\right)^{2}}{1024 \omega_{k}^{12}\left(\tau_{k i n}\right)}$.

Therefore, deriving the Raychaudury equation twice, one has $\dddot{H}=-\frac{1}{M_{p l}^{2}}\left(\ddot{\varphi}^{2}+\dot{\varphi} \dddot{\varphi}\right)$, obtaining

$$
\begin{aligned}
& \dddot{H}\left(\tau_{k i n}^{-}\right)-\dddot{H}\left(\tau_{k i n}^{+}\right)= \\
& -\frac{1}{M_{p l}^{2}} \dot{\varphi}_{k i n}\left(\dddot{\varphi}\left(\tau_{k i n}^{-}\right)-\dddot{\varphi}\left(\tau_{k i n}^{+}\right)\right) .
\end{aligned}
$$

In addition, from the temporal derivative of the conservation equation, $\dddot{\varphi}+3 \dot{H} \dot{\varphi}+3 H \ddot{\varphi}+\dot{\varphi} V_{\varphi \varphi}=0$, and taking into account that $V_{\varphi \varphi}\left(0^{+}\right)=0$, one deduces that for the model (2)

$$
\begin{aligned}
& \dddot{\varphi}\left(\tau_{\text {kin }}^{-}\right)-\dddot{\varphi}\left(\tau_{k i n}^{+}\right)=-\dot{\varphi}_{k i n} V_{\varphi \varphi}\left(0^{-}\right) \\
& \quad=-\frac{4}{3} \lambda \dot{\varphi}_{k i n} M_{p l}^{2},
\end{aligned}
$$

thus, using (20) and (21), one gets

$\dddot{H}\left(\tau_{\text {kin }}^{-}\right)-\dddot{H}\left(\tau_{\text {kin }}^{+}\right)=\frac{4}{3} \lambda \dot{\varphi}_{\text {kin }}^{2}$

and the expression of the square of the $\beta_{k}$-Bogoliubov coefficient becomes

$\left|\beta_{k}\right|^{2} \cong \frac{m_{A}^{4} \lambda^{2} a_{k i n}^{12} \dot{\varphi}_{k i n}^{4}}{576 \omega_{k}^{12}\left(\tau_{k i n}\right)}$.

On the other hand, the energy density of the produced particles $\rho_{A}(\tau)=\frac{1}{2 \pi^{2} a^{4}(\tau)} \int_{0}^{\infty} \omega_{k}(\tau) k^{2}\left|\beta_{k}\right|^{2} d k \quad$ (see for instance [28]), before the decay of the $X$-particles, evolves as

$$
\begin{aligned}
\rho_{A}(\tau) & \cong \frac{m_{A}}{2 \pi^{2} a^{3}(\tau)} \int_{0}^{\infty} k^{2}\left|\beta_{k}\right|^{2} d k \\
& \cong 3.7 \times 10^{-6} \lambda^{2}\left(\frac{\dot{\varphi}_{k i n}}{m_{A}}\right)^{4}\left(\frac{a_{k i n}}{a(\tau)}\right)^{3},
\end{aligned}
$$

where $A=X, Y$.

Remark 3.1 Note that creation of superheavy particles in this model is power law small. Effectively, $\rho_{A}\left(\tau_{k i n}\right) \sim\left(\frac{\dot{\varphi}_{k i n}}{m_{A}}\right)^{4}$ and this is due to the discontinuity of the second derivative of the potential at $\varphi=0$. On the contrary, when the potential is very smooth the energy density of the created superheavy particles is exponentially suppressed by a factor $e^{-c_{A} m_{A} / H_{k i n}}$ [9], where $c_{A}$ is a model-dependent dimensionless parameter, meaning that for such a class of potentials the gravitational particle production mechanism is not efficient.

Thus, before the decay of the $X$-particles, one will have

$\rho_{Y}(\tau)=\left(\frac{m_{X}}{m_{Y}}\right)^{4} \rho_{X}(\tau)$,

which means that, for the PV model, one has to assume $m_{X} \ll$ $m_{Y}$ in order to have a radiation era.

At this point, it is important to take into account that when reheating is due to the gravitational production of superheavy particles, in order that the overproduction of GWs does not alter the BBN success, the decay of these particles has to take place after the end of kination [26]. Then, assuming as 
usual instantaneous thermalization, the reheating is produced immediately after the decay of the $X$-particles, obtaining

$\rho_{Y, r h}=\left(\frac{m_{X}}{m_{Y}}\right)^{4} \rho_{X, r h}$,

where, the subindex "rh" means that the quantities are evaluated at the reheating time. After reheating, the evolution of the corresponding energy densities will be

$\rho_{X}(\tau)=\rho_{X, r h}\left(\frac{a_{r h}}{a(\tau)}\right)^{4}, \quad \rho_{Y}(\tau)=\rho_{Y, r h}\left(\frac{a_{r h}}{a(\tau)}\right)^{3}$,

meaning that at the matter-radiation equality:

$\frac{a_{r h}}{a_{e q}}=\frac{\rho_{Y, r h}}{\rho_{X, r h}}=\left(\frac{m_{X}}{m_{Y}}\right)^{4}$,

and consequently

$\rho_{Y, e q}=\rho_{Y, r h}\left(\frac{m_{X}}{m_{Y}}\right)^{12}=\frac{\pi^{2} g_{*}}{30} T_{r h}^{4}\left(\frac{m_{X}}{m_{Y}}\right)^{16}$,

where $T_{r h}$ is the reheating temperature and $g_{*}=106.75$ are the degrees of freedom for the Standard Model.

On the other hand, considering the central values obtained in [30] of the red shift at the matter-radiation equality $z_{e q}=3365$, the present value of the ratio of the matter energy density to the critical one $\Omega_{m, 0}=0.308$, and $H_{0}=67.81 \mathrm{Km} / \mathrm{sec} / \mathrm{Mpc}$, one can deduce that the present value of the matter energy density is $\rho_{m, 0}=3 H_{0}^{2} M_{p l}^{2} \Omega_{m, 0}=$ $3.26 \times 10^{-121} M_{p l}^{4}$, and at matter-radiation equality one will have $\rho_{m, e q}=\rho_{m, 0}\left(1+z_{e q}\right)^{3}=4.4 \times 10^{-1} \mathrm{eV}^{4}$. Since practically all the matter has a not baryonic origin, one can conclude that $\rho_{Y, e q} \cong \rho_{m, e q}$, meaning that the reheating temperature is given by a function of $m_{Y} / m_{X}$ as follows:

$T_{r h} \cong 3.3 \times 10^{-10}\left(\frac{m_{Y}}{m_{X}}\right)^{4} \mathrm{GeV}$.

\subsection{Decay after the end of the kination regime}

As we have already explained in the previous Section, in order that the overproduction of GWs does not alter the BBN success, the decay of the $X$-particles has to be produced after the end of kination, which occurs when the energy density of the inflaton field is equal to the one of the $X$-particles, i.e., when $\rho_{X}\left(\tau_{\text {end }}\right)=\rho_{\varphi}\left(\tau_{\text {end }}\right)$, where we have denoted by $\tau_{\text {end }}$ the time at which kination ends. Then, the decaying rate, namely $\Gamma$, has to satisfy $\Gamma \leq H\left(\tau_{\text {end }}\right) \equiv H_{\text {end }}$, and one has

$H_{\text {end }}^{2}=\frac{2 \rho_{\varphi, \text { end }}}{3 M_{p l}^{2}}$

and

$\rho_{\varphi, \text { end }}=\rho_{\varphi, k i n}\left(\frac{a_{k i n}}{a_{\text {end }}}\right)^{6}=3 H_{k i n}^{2} M_{p l}^{2}\left(\frac{a_{k i n}}{a_{\text {end }}}\right)^{6}$.
Now, taking into account that during kination the energy density of the inflaton field decays as $a^{-6}$, and the one of the produced particles as $a^{-3}$, at the end ok kination $\left(\rho_{X \text {, end }}=\right.$ $\left.\rho_{\varphi, \text { end }}\right)$, we will have

$\rho_{X, k i n}\left(\frac{a_{k i n}}{a_{\text {end }}}\right)^{3}=\rho_{\varphi, k i n}\left(\frac{a_{k i n}}{a_{\text {end }}}\right)^{6}$,

that is, $\left(\frac{a_{k i n}}{a_{\text {end }}}\right)^{3}=\frac{\rho_{X, k i n}}{\rho_{\varphi, k i n}}$, and introducing the so-called heating efficiency defined in [31] as

$\Theta \equiv \frac{\rho_{X, k i n}}{\rho_{\varphi, k i n}} \cong 7.2 \times 10^{-38}\left(\frac{M_{p l}}{m_{X}}\right)^{4}$,

we can write $\rho_{\varphi, \text { end }}=3 H_{k i n}^{2} M_{p l}^{2} \Theta^{2}$.

Consequently, (31) leads to $H_{\text {end }}=\sqrt{2} H_{k i n} \Theta$, and from the constraint $\Gamma \leq H_{\text {end }}$ one obtains the bound

$\frac{\Gamma}{M_{p l}} \leq 1.5 \times 10^{-43}\left(\frac{M_{p l}}{m_{X}}\right)^{4}$.

On the other hand, assuming once again instantaneous thermalization, the energy density of the $X$-particles at the reheating time will be $\rho_{X, r h}=3 \Gamma^{2} M_{p l}^{2}$, and thus, the reheating temperature will be given by:

$T_{r h}=\left(\frac{90}{\pi^{2} g_{*}}\right)^{\frac{1}{4}} \sqrt{\Gamma M_{p l}} \cong 1.3 \times 10^{18} \sqrt{\frac{\Gamma}{M_{p l}}} \mathrm{GeV}$.

As a consequence, from the two expressions of the reheating temperature (30) and (36) one can write the mass of the dark matter as a function of $\Gamma$ and $m_{X}$ as follows:

$m_{Y} \cong 7.9 \times 10^{6}\left(\frac{\Gamma}{M_{p l}}\right)^{1 / 8} m_{X}$.

Remark 3.2 In our work we have not considered the production of light particles nearly conformally coupled with gravity [3] because its energy never dominates and do not have any influence in the evolution of the Universe. Effectively, the energy density of these light particles, namely $\rho_{r}$, evolves as [1,3] (see also [32] for a detailed discussion)

$\rho_{r}(\tau) \cong 10^{-2}(1-6 \xi)^{2} H_{k i n}^{4}\left(\frac{a_{k i n}}{a(\tau)}\right)^{4}$,

where $\xi$ is the coupling constant and for the sake of simplicity we will take $|1-6 \xi| \sim 10^{-2}$, although it could be smaller than $10^{-2}$.

Then, when the energy density of the $X$-particles is of the same order than of the field $\varphi$, one has $\rho_{r, \text { end }} \cong$ $10^{-6} H_{k i n}^{4} \Theta^{4 / 3}$ which has to be compared with $\rho_{\varphi, \text { end }}=$ $3 H_{k i n}^{2} M_{p l}^{2} \Theta^{2}$. A simple calculation leads to

$\frac{\rho_{r, \text { end }}}{\rho_{\varphi, \text { end }}} \cong 1.3 \times 10^{-3}\left(10^{14} \frac{m_{X}^{4}}{M_{p l}^{4}}\right)^{2 / 3}$ 
and for masses satisfying $m_{X} \leq 3 \times 10^{-3} M_{p l} \cong 7.3 \times$ $10^{15} \mathrm{GeV}$, which as we will see enter in our range, we have

$$
\frac{\rho_{r, \text { end }}}{\rho_{\varphi, \text { end }}} \leq 0.53 \text {, }
$$

concluding that the energy density of the light particles created during the phase transition from the end of inflation to the beginning of kination never dominates because its energy density decreases as $a^{-4}$ while the one of $X$-particles as $a^{-3}$.

\subsection{Overproduction of GWs}

The success of the BBN demands that the ratio of the energy density of GWs to the one of the produced particles at the reheating time satisfies [33]

$$
\frac{\rho_{G W, r h}}{\rho_{X, r h}} \leq 10^{-2},
$$

where the energy density of the GWs is given by $\rho_{G W}(\tau) \cong$ $10^{-2} H_{k i n}^{4}\left(a_{k i n} / a(\tau)\right)^{4}$ (see for instance [3]).

Therefore, taking into account that

$$
\left(\frac{a_{k i n}}{a_{\text {end }}}\right)^{4}=\Theta^{4 / 3}
$$

and

$$
\left(\frac{a_{\text {end }}}{a_{r h}}\right)^{4}=\left(\frac{\rho_{X, r h}}{\rho_{X, \text { end }}}\right)^{4 / 3}=\left(\frac{\Gamma}{\sqrt{2} H_{k i n} \Theta}\right)^{8 / 3},
$$

writing $\frac{a_{k i n}}{a_{r h}}=\left(\frac{a_{k i n}}{a_{\text {end }}}\right)\left(\frac{a_{\text {end }}}{a_{r h}}\right)$ we will have

$\rho_{G W, r h}=10^{-2} H_{k i n}^{4}\left(\frac{\Gamma}{\sqrt{2 \Theta} H_{k i n}}\right)^{8 / 3}$,

and thus,

$\frac{\rho_{G W, r h}}{\rho_{X, r h}} \cong 7.2 \times 10^{38}\left(\frac{m_{X}}{M_{p l}}\right)^{16 / 3}\left(\frac{\Gamma}{M_{p l}}\right)^{2 / 3}$,

meaning that the bound (41) leads to the constraint

$$
\frac{\Gamma}{M_{p l}} \leq 5.1 \times 10^{-62}\left(\frac{M_{p l}}{m_{X}}\right)^{8} .
$$

Here, it is important to realize that for $m_{X} \geq 2.4 \times$ $10^{-5} M_{p l}$ the constraint (46) automatically implies (35), and thus, taking into account that $T_{r h}>1 \mathrm{MeV}$ because the BBN occurs at the $\mathrm{MeV}$ regime [34], one gets that $\Gamma$ must satisfy

$$
5.9 \times 10^{-43} \leq \frac{\Gamma}{M_{p l}} \leq 5.1 \times 10^{-62}\left(\frac{M_{p l}}{m_{X}}\right)^{8}
$$

which always holds when

$$
5.8 \times 10^{13} \mathrm{GeV} \leq m_{X} \leq 10^{16} \mathrm{GeV} .
$$

Taking into account that $m_{X} \geq 10^{15} \mathrm{GeV}$, (recall that, as we have explained in Sect. $2, m_{X} \gg H_{B} \sim 10^{14} \mathrm{GeV}$ ) the mass of $X$-particles is constrained to $10^{15} \mathrm{GeV} \leq m_{X} \leq$
$10^{16} \mathrm{GeV}$, and consequently, from (36) and (47), for our model the reheating temperature is bounded by

$1 \mathrm{MeV} \leq T_{r h} \leq 9.7 \mathrm{GeV}$,

and from (37) and (47) the mass of the $Y$-particles by

$4.1 \times 10^{16} \mathrm{GeV} \leq m_{Y} \leq 4.1 \times 10^{17} \mathrm{GeV}$.

We finish this Section with the following remark: As we can see, the choice of masses of the $X$-field greater than $10^{15} \mathrm{GeV}$ produce a very low reheating temperature. However, as has been discussed in the introduction of [18] (see also the end of the Section 4.2 in [17] and the bound obtained in [31]), when reheating is via gravitational production of light particles, for very low temperatures less than $10^{4} \mathrm{GeV}$, a spike in the Gravitational Wave spectrum, which is large enough to challenge the BBN process, is generated during kination. To overpass this situation we have to consider masses of the $X$-field satisfying the condition $5.8 \times 10^{13} \leq m_{X}<10^{14} \mathrm{GeV}$, because in this situation, taking $\frac{\Gamma}{M_{p l}}=5.1 \times 10^{-62}\left(\frac{M_{p l}}{m_{X}}\right)^{8}$ in (47), one gets

$T_{r h}=2.93 \times 10^{-13}\left(\frac{M_{p l}}{m_{X}}\right)^{4} \mathrm{GeV}$,

which for $m_{X}<10^{14} \mathrm{GeV}$, leads to the lower bound $T_{r h} \geq$ $10^{4} \mathrm{GeV}$.

Another way to alleviate this situation is to assume that the $X$-field is not conformally coupled with gravity. In this situation, the $X$-field could have masses of the order $10^{15} \mathrm{GeV}$ or greater, obtaining a maximum reheating temperature of 66 $\mathrm{TeV}$ (see [37] for a detailed discussion).

Finally, as we will see in next Section, when the particles responsible for the reheating are created via instant preheating this problem disappear, because the reheating temperature is around $10^{8} \mathrm{GeV}$.

\section{Instant preheating}

In this Section we consider an interaction between the scalar field and a massless $X$-field conformally coupled with gravity, whose interacting Lagrangian is given by $\mathcal{L}_{i n t}=$ $-\frac{1}{2} g^{2} \varphi^{2} X^{2}$, where $g$ is a coupling constant and the enhanced symmetry point has been chosen $\varphi=0$, because, as we have already seen, at this point the velocity of the scalar field is nearly maximum (see Fig. 2), what, as one can see from formula (57), maximizes the particle production. In this situation $X$-particles, having an effective mass $m_{\text {eff }}=g \varphi(t)$, are created via a mechanism named instant preheating, which was introduced in [13] in the framework of standard inflation, and was applied, for the first time, to quintessential inflation in [35]. 
Remark 4.1 Note that here the $X$-field is completely different to the one considered in the previous Sections, however the superheavy dark matter $Y$-field is the same, i.e., it continues only interacting gravitationally.

As was discussed in [35], in order to avoid a second inflationary period, it is mandatory that, unlike the superheavy particles created gravitationally studied in the previous section, these $X$-particles decay well before the end of kination. Then, at the matter-radiation equality we will have

$\rho_{X, e q}=\rho_{X, d e c}\left(\frac{a_{d e c}}{a_{e q}}\right)^{4}, \quad \rho_{Y, e q}=\rho_{Y, d e c}\left(\frac{a_{d e c}}{a_{e q}}\right)^{3}$,

and since $\rho_{X, e q}=\rho_{Y, e q}$ one will have

$\rho_{Y, e q}=\rho_{Y, d e c}\left(\frac{\rho_{Y, d e c}}{\rho_{X, d e c}}\right)^{3}$.

Now, using that the decay of the $X$-particles is finished when $\Gamma=H_{d e c}=H_{k i n}\left(\frac{a_{k i n}}{a_{d e c}}\right)^{3}$, and that the energy density of the $Y$-particles decreases as $a^{-3}$, i.e., $\rho_{Y, d e c}=$ $\rho_{Y, k i n}\left(\frac{a_{k i n}}{a_{d e c}}\right)^{3}$, we obtain

$\rho_{Y, e q}=\rho_{Y, k i n} \frac{\Gamma}{H_{k i n}}\left(\frac{\rho_{Y, d e c}}{\rho_{X, d e c}}\right)^{3}$.

In addition, taking into account that at the decay time the scalar field is near $M_{p l}$ (see for details [35]), and thus, the effective mass of the $X$-particles is $g M_{p l}$, one gets

$\rho_{X, d e c}=g M_{p l} n_{X, d e c}=g M_{p l} n_{X, k i n}\left(\frac{a_{k i n}}{a_{d e c}}\right)^{3}$,

where $n_{X}$ denotes the number density of produced $X$ particles.

Therefore, one will have

$\frac{\rho_{Y, d e c}}{\rho_{X, d e c}}=\frac{\rho_{Y, k i n}}{g M_{p l} n_{X, k i n}}$.

On the other hand, at the beginning of kination the number density of $X$-particles is [35]

$n_{X, k i n}=\frac{g^{3 / 2} \dot{\varphi}_{k i n}^{3 / 2}}{8 \pi^{3}}$,

and the energy density of the $Y$-particles is given by the formula (24), meaning that, at the matter-radiation equality one has

$$
\begin{aligned}
\rho_{Y, e q} & =\rho_{Y, k i n} \frac{\Gamma}{H_{k i n}}\left(\frac{8 \pi^{3} \rho_{Y, k i n}}{g^{5 / 2} M_{p l} \dot{\varphi}_{k i n}^{3 / 2}}\right)^{3} \\
& \cong 9.6 \times 10^{-53} g^{-15 / 2}\left(\frac{M_{p l}}{m_{Y}}\right)^{16} \frac{\Gamma}{M_{p l}} \mathrm{eV}^{4},
\end{aligned}
$$

which compared with the observational value of the matter density at the matter-radiation equality $4.4 \times 10^{-1} \mathrm{eV}^{4}$, leads to
$m_{Y} \cong 5.9 \times 10^{-4} g^{-15 / 32}\left(\frac{\Gamma}{M_{p l}}\right)^{1 / 16} M_{p l}$.

Dealing with the reheating temperature, if one assumes once again instantaneous thermalization, it is given by (see [36] for details)

$$
\begin{aligned}
T_{r h} & =\left(\frac{30}{g_{*} \pi^{2}}\right)^{1 / 4} \rho_{X, \operatorname{dec}}^{1 / 4} \sqrt{\frac{\rho_{X, d e c}}{\rho_{\varphi, d e c}}} \\
& \cong 10^{14} g^{15 / 8}\left(\frac{M_{p l}}{\Gamma}\right)^{1 / 4} \mathrm{GeV},
\end{aligned}
$$

because at the end of the decay of the $X$-particles

$$
\begin{aligned}
& \rho_{\varphi, d e c}=3 \Gamma^{2} M_{p l}^{2} \text { and } \\
& \rho_{X, d e c} \cong 10^{-2} g^{5 / 2} \sqrt{\frac{H_{k i n}}{M_{p l}}} \frac{\Gamma}{M_{p l}} M_{p l}^{4} .
\end{aligned}
$$

When $X$-particles decay into fermions via a Yukawa type interaction $h \psi \bar{\psi} X$ with a decaying rate $\Gamma=\frac{h^{2} g M_{p l}}{8 \pi}$, where $h$ is a coupling constant [35], the mass of the $Y$-particles and the reheating temperature become

$m_{Y} \cong 1.1 \times 10^{15} g^{-13 / 32} h^{1 / 8} \mathrm{GeV}$ and

$T_{r h} \cong 2.2 \times 10^{14} g^{13 / 8} h^{-1 / 2} \mathrm{GeV}$.

However, as has been showed in [36] there is a narrow range of values of the parameters $g$ and $h$ for which instant preheating is viable. For example, choosing $\left(h=10^{-1}, g=\right.$ $\left.10^{-4}\right)$ or $\left(h=10^{-2}, g=5 \times 10^{-5}\right)$ one gets:

$m_{Y} \sim 10^{16} \mathrm{GeV}$ and $T_{r h} \sim 2.2 \times 10^{8} \mathrm{GeV}$.

Finally, we want to stress that when the particle production of $X$-particles is via instant preheating the overproduction of GWs does not alter the success of the BBN, because

$\frac{\rho_{G W, r h}}{\rho_{X, r h}} \leq \frac{\rho_{G W, k i n}}{g M_{p l} n_{X, k i n}} \cong 1.6 \times 10^{-16} g^{-5 / 2} \leq 10^{-5}$.

\section{Evolution of the universe in quintessential inflation}

This section is a review of [14] and the Section 4 of [37].

We start with the initial conditions, at the beginning of kination for the model (2), obtained in Sect. 2:

$\varphi_{k i n}=0, \quad \dot{\varphi}_{k i n}=3.54 \times 10^{-6} M_{p l}^{2}$.

During kination, the scale factor and the Hubble rate evolves as $a \propto t^{1 / 3} \Longrightarrow H=\frac{1}{3 t}$, and from the Friedmann equation, the evolution in this phase will be

$$
\frac{\dot{\varphi}^{2}}{2}=\frac{M_{p l}^{2}}{3 t^{2}} \Longrightarrow \varphi(t)=\sqrt{\frac{2}{3}} M_{p l} \ln \left(\frac{H_{k i n}}{H(t)}\right) .
$$


Then, at the end of kination, one has

$\varphi_{\text {end }}=-\sqrt{\frac{2}{3}} M_{p l} \ln (\sqrt{2} \Theta), \dot{\varphi}_{\text {end }}=2 \sqrt{3} M_{p l} H_{k i n} \Theta$,

where, once again, we have used the relation $H_{\text {end }}=$ $\sqrt{2} H_{k i n} \Theta$.

During the period between $t_{\text {end }}$ and $t_{r h}$, in the case that the $X$ particles are created gravitationally, the universe is matter dominated and, thus, the Hubble parameter becomes $H=\frac{2}{3 t}$. During this epoch, the gradient of the potential could also be disregarded, hence, the equation of the scalar field becomes $\ddot{\varphi}+\frac{2}{t} \dot{\varphi}=0$, and thus, after few calculations, at the reheating time one has

$\varphi_{r h} \cong \varphi_{\text {end }}+\sqrt{\frac{2}{3}} M_{p l}$

and

$\dot{\varphi}_{r h}=\frac{\sqrt{3}}{4} \frac{M_{p l} H_{r h}^{2}}{H_{k i n} \Theta}$

During the radiation period one can continue disregarding the potential, obtaining

$\varphi(t)=\varphi_{r h}+2 \dot{\varphi}_{r h} t_{t r}\left(1-\sqrt{\frac{t_{r h}}{t}}\right)$,

and thus, since $\dot{\varphi}_{r h} t_{t r}=\frac{\pi}{6} \sqrt{\frac{g_{*}}{30}} \frac{T_{r h}^{2}}{H_{k i n} \Theta}$ (in [14] and [37] wrongly the authors take $\dot{\varphi}_{r h} t_{t r}=\sqrt{\frac{2}{3}} M_{p l}$ ) at the matterradiation equality one has

$$
\begin{aligned}
\varphi_{e q} & =\varphi_{r h}+\frac{\pi}{3} \sqrt{\frac{g_{*}}{30}} \frac{T_{r h}^{2}}{H_{k i n} \Theta}\left(1-\sqrt{\frac{4 H_{e q}}{3 H_{r h}}}\right) \\
& =\varphi_{r h}+\frac{\pi}{3} \sqrt{\frac{g_{*}}{30}} \frac{T_{r h}^{2}}{H_{k i n} \Theta}\left(1-\sqrt{\frac{4}{3}}\left(\frac{g_{e q}}{g_{*}}\right)^{1 / 4} \frac{T_{e q}}{T_{r h}}\right) \\
& \cong \varphi_{r h}+\frac{\pi}{3} \sqrt{\frac{g_{*}}{30}} \frac{T_{r h}^{2}}{H_{k i n} \Theta} \cong \varphi_{r h}+\frac{2 T_{r h}^{2}}{H_{k i n} \Theta},
\end{aligned}
$$

where $g_{e q} \cong 3.36$ are the degrees of freedom at the matterradiation equality and $T_{e q}$ is the temperature of the radiation at the matter-radiation equilibrium, which is related with the energy density via the relation $\rho_{e q}=\frac{\pi^{2}}{15} g_{e q} T_{e q}^{4} \cong 8.8 \times$ $10^{-1} \mathrm{eV}^{4}$, and thus, given by $T_{e q} \cong 7.9 \times 10^{-10} \mathrm{GeV}$.

In the same way,

$$
\begin{aligned}
\dot{\varphi}_{e q} & =\dot{\varphi}_{r h} \frac{t_{r h}}{t_{e q}} \sqrt{\frac{t_{r h}}{t_{e q}}}=\left(\frac{16 g_{e q}}{9 g_{*}}\right)^{3 / 4}\left(\frac{T_{e q}}{T_{r h}}\right)^{3} \dot{\varphi}_{r h} \\
& \cong 1.7 \frac{T_{e q}^{3} T_{r h}}{M_{p l} H_{k i n} \Theta} \cong 5.8 \times 10^{-46} \frac{T_{r h} m_{X}^{4}}{M_{p l}^{3}} .
\end{aligned}
$$

After the matter-radiation equality the dynamical equations can not be solved analytically and, thus, one needs to use numerics to compute them. In order to do that, we need to use a "time" variable that we choose to be minus the number of $e$-folds up to the present epoch, namely, $N \equiv-\ln (1+z)=\ln \left(\frac{a}{a_{0}}\right)$. Now, using the variable $N$, one can recast the energy density of radiation (the energy density of the decay products of the $X$-field which we continue denoting by $\rho_{X}$ ) and dark matter respectively as

$\rho_{X}(N)=\frac{\rho_{e q}}{2} e^{4\left(N_{e q}-N\right)}, \quad \rho_{Y}(N)=\frac{\rho_{e q}}{2} e^{3\left(N_{e q}-N\right)}$,

where $N_{e q}=-\ln \left(1+z_{e q}\right)=-8.121$ is the value of $N$ at the matter-radiation equality.

In order to obtain the dynamical system for the (2) model, we introduce the following dimensionless variables

$x=\frac{\varphi}{M_{p l}}, \quad y=\frac{\dot{\varphi}}{H_{0} M_{p l}}$,

where $H_{0} \cong 1.42 \times 10^{-33} \mathrm{eV}$ denotes the current value of the Hubble parameter. Now, using the variable $N=-\ln (1+z)$ defined above and also using the conservation equation $\ddot{\varphi}+$ $3 H \dot{\varphi}+V_{\varphi}=0$, we will have the following non-autonomous dynamical system:

$\left\{\begin{array}{l}x^{\prime}=\frac{y}{\bar{H}} \\ y^{\prime}=-3 y-\frac{\bar{V}_{x}}{\bar{H}}\end{array}\right.$

where the prime represents the derivative with respect to $N$, $\bar{H}=\frac{H}{H_{0}}$ and $\bar{V}=\frac{V}{H_{0}^{2} M_{p l}^{2}}$. Moreover, the Friedmann equation now looks as

$\bar{H}(N)=\frac{1}{\sqrt{3}} \sqrt{\frac{y^{2}}{2}+\bar{V}(x)+\bar{\rho}_{X}(N)+\bar{\rho}_{Y}(N),}$

where we have introduced the following dimensionless energy densities $\bar{\rho}_{X}=\frac{\rho_{X}}{H_{0}^{2} M_{p l}^{2}}$ and $\bar{\rho}_{Y}=\frac{\rho_{Y}}{H_{0}^{2} M_{p l}^{2}}$.

Then, we have to integrate the dynamical system, starting at $N_{e q}=-8.121$, with initial condition $x_{e q}$ and $y_{e q}$ which are obtained analytically in formulas (71) and (72). The value of the parameter $\tilde{M}$ is obtained equaling at $N=0$ the equation (76) to 1 , i.e., imposing $\bar{H}(0)=1$.

On the other hand, note that

$y_{e q}=4.1 \times 10^{-4} \frac{T_{r h}}{\mathrm{GeV}}\left(\frac{m_{X}}{M_{p l}}\right)^{4}$,

and thus, for viable reheating temperatures $T_{r h} \leq 9.7 \mathrm{GeV}$ one has $y_{e q} \ll 1$. And for $x_{e q}$, after a simple calculation, one gets (Fig. 4) 


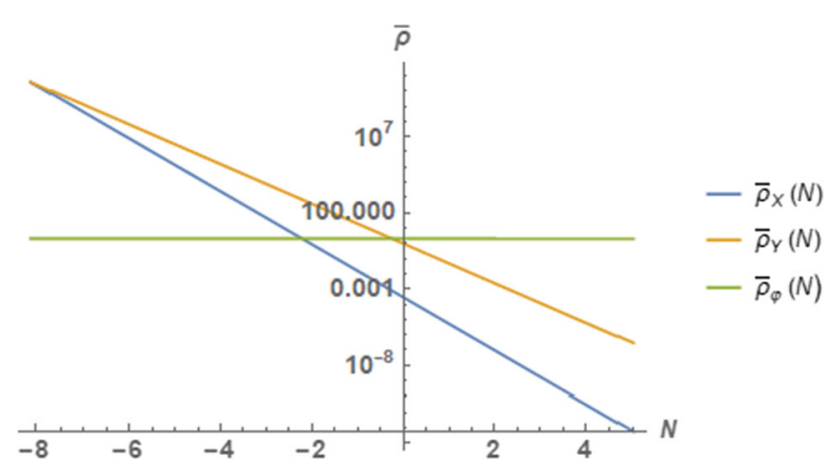

Fig. 3 Evolution of the different dimensionless energy densities for $m_{X} \sim 10^{14} \mathrm{GeV}$ and $T_{r h} \sim 10^{4} \mathrm{GeV}$

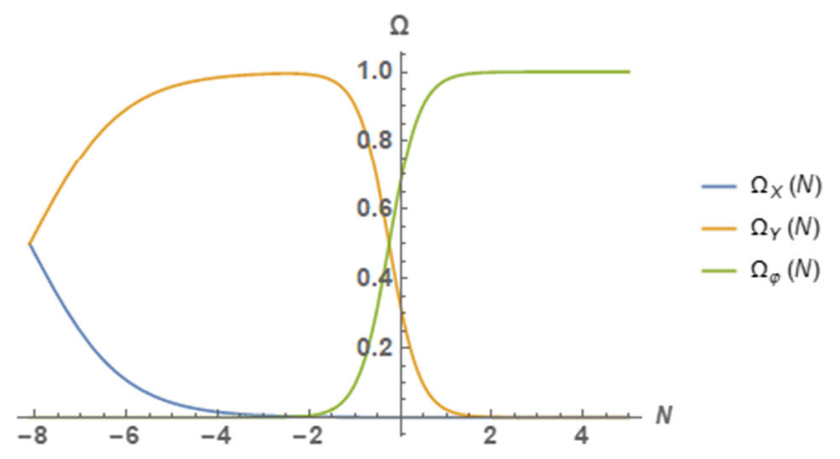

Fig. 4 Evolution of the different $\Omega$ for $m_{X} \sim 10^{14} \mathrm{GeV}$ and $T_{r h} \sim$ $10^{4} \mathrm{GeV}$. At late times $\Omega_{\varphi}=1$, meaning that all the energy density of the universe is the one of the scalar field

$$
\begin{aligned}
x_{e q} \cong & \sqrt{\frac{2}{3}}\left(86.17-4 \ln \left(\frac{M_{p l}}{m_{X}}\right)\right) \\
& +1.9 \times 10^{41} \frac{T_{r h}^{2}}{M_{p l}^{2}}\left(\frac{m_{X}}{M_{p l}}\right)^{4} .
\end{aligned}
$$

Summing up, what we have obtained numerically for the viable values of the reheating temperature and the masses of the $X$-filed is that the value of the mass $\tilde{M}$ ranges between $2.5 \times 10^{5}$ and $8.6 \times 10^{5} \mathrm{GeV}$, what completely agrees with the value obtained by Peebles and Vilenkin in his seminal paper [1]. In addition, as one can see in Fig. 3 that the scalar field slow-rolls the inverse power law potential after the matterradiation equality, leading to an eternal acceleration because the effective Equation of State parameter goes towards -1 (see Fig. 5).

Finally note that the for the potential (2) the energy scale of inflation is [38] $V^{1 / 4}\left(\varphi \ll-M_{p l}\right) \sim \lambda^{1 / 4} M_{p l} \sim 10^{15} \mathrm{GeV}$, which is very close to the GUT scales, while the energy scale for Dark Energy $V^{1 / 4}(\varphi \cong 0) \sim \lambda^{1 / 4} \tilde{M} \sim 10^{2} \mathrm{GeV}$ is near the electroweak scale. Therefore, our model provides natural scales for inflation and Dark Energy.

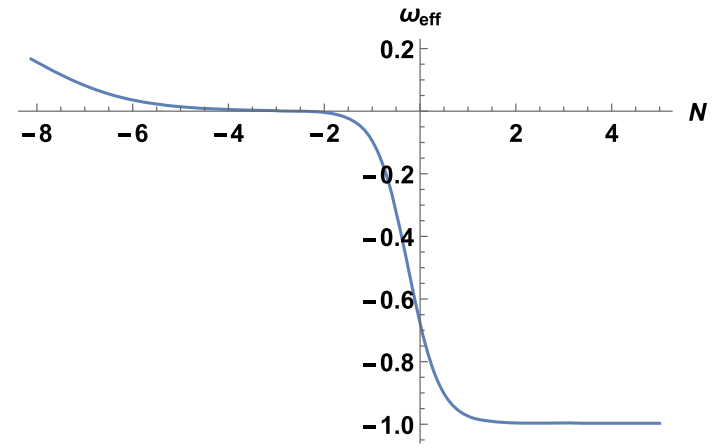

Fig. 5 Evolution of the effective Equation of State parameter for $m_{X} \sim$ $10^{14} \mathrm{GeV}$ and $T_{r h} \sim 10^{4} \mathrm{GeV}$. Al late times $w_{\text {eff }} \rightarrow-1$, what means that the universe accelerates forever entering in a de Sitter phase

\section{Conclusions}

In this paper we have presented the idea of creating dark matter in a quintessential inflation model whose potential, which is an improvement of the well-known Peebles-Vilenkin one, is composed by a Starobinsky Inflationary type-potential matched with an inverse power law potential, which is responsible for quintessence. Since the phase transition from the end of inflation to the beginning of kination is very abrupt, the adiabatic regime is broken and superheavy particles could be gravitationally produced. We have assumed two different reheating mechanisms:

1. In the first one, two kind of superheavy particles are gravitationally produced. $X$-particles, whose decay products form the baryonic matter, and GIMP $Y$-particles, which are responsible for the dark matter abundance. For this model we have shown that, for reasonable masses of the $X$-particles between $10^{14}$ and $10^{16} \mathrm{GeV}$, a viable model with a reheating temperature from the $\mathrm{MeV}$ to the $\mathrm{TeV}$ regime is obtained when the mass of the dark matter particles is of the order $10^{16}-10^{17} \mathrm{GeV}$.

2. The second mechanism is the well known instant preheating, where now the $X$-field is massless and coupled with the scalar field, and the superheavy $Y$-field depicting dark matter continues only interacting gravitationally. In this situation, a viable model requires a reheating temperature around $10^{8} \mathrm{GeV}$ and dark matter particles with masses around $10^{16} \mathrm{GeV}$.

Finally, in the case that both kind of particles are produced gravitationally, we have shown numerically that the model leads, at late times, to an eternal inflation with and Equation of State parameter equal to -1 .

Acknowledgements I would like to thank my colleague Llibert Aresté Saló for his valuable help in doing the numerical calculations, to my other colleague Jaume Amorós for reading all the previous versions 
of the manuscript, and also to the referees for your suggestions that have been essential to improve this work. This investigation has been supported by MINECO (Spain) grant MTM2017-84214-C2-1-P, and in part by the Catalan Government 2017-SGR-247.

Data Availability Statement This manuscript has associated data in a data repository. [Authors' comment: Data have been uploaded in arXiv:1904.02393[gr-qc].]

Open Access This article is licensed under a Creative Commons Attribution 4.0 International License, which permits use, sharing, adaptation, distribution and reproduction in any medium or format, as long as you give appropriate credit to the original author(s) and the source, provide a link to the Creative Commons licence, and indicate if changes were made. The images or other third party material in this article are included in the article's Creative Commons licence, unless indicated otherwise in a credit line to the material. If material is not included in the article's Creative Commons licence and your intended use is not permitted by statutory regulation or exceeds the permitted use, you will need to obtain permission directly from the copyright holder. To view a copy of this licence, visit http://creativecomm ons.org/licenses/by/4.0/.

Funded by SCOAP ${ }^{3}$.

\section{References}

1. P.J.E. Peebles, A. Vilenkin, Phys. Rev. D 59, 063505 (1999). arXiv:hep-ph/9810509

2. K. Dimopoulos, J.W.F. Valle, Astropart. Phys. 18, 287 (2002). arXiv:astro-ph/0111417

3. L.H. Ford, Phys. Rev. D 35, 2955 (1987)

4. T. Damour, A. Vilenkin, Phys. Rev. D 53, 2981 (1995). arXiv:hep-th/9503149

5. S. Hashiba, J. Yokoyama, JCAP 01, 028 (2019). arXiv: 1809.05410

6. S. Hashiba, J. Yokoyama, Phys. Rev. D 99, 043008 (2019). arXiv: 1812.10032

7. D.J.H. Chung, P. Crotty, E.W. Kolb, A. Riotto, Phys. Rev. D 64, 043503 (2001). arXiv:hep-ph/0104100

8. Y. Ema, K. Nakayama, Y. Tang, JHEP 09, 135 (2018). arXiv: 1804.07471

9. D.J.H. Chung, E.W. Kolb, A.J. Long, JHEP 01, 189 (2019). arXiv: 1812.00211

10. A.A. Starobinsky, Phys. Lett. B 91, 99 (1980)

11. A.A. Starobinsky, Proc. of the Second Seminar "Quantum Theory of Gravity", INR Press, Moscow 58-72 (1982)

12. A. Vilenkin, Phys. Rev. D 32, 2511 (1985)
13. G. Felder, L. Kofman, A. Linde, Phys. Rev. D 59, 123523 (1999). arXiv:hep-ph/9812289

14. J. Haro, J. Amorós, S. Pan, Eur. Phys. J. C 79(6), 505 (2019). arXiv: 1901.00167

15. N. Aghanim et al. Planck 2018 results. VI. Cosmological parameters (2018). arXiv: 1807.06209

16. C.Q. Geng, C.C. Lee, M. Sami, E.N. Saridakis, A.A. Starobinsky, JCAP 1706(06), 011 (2017). arXiv:1705.01329

17. K. Dimopoulos, C. Owen, JCAP 1706, 027 (2017). arXiv:1703.00305

18. K. Dimopoulos, L. Donaldson Wood, C. Owen, Phys. Rev. D 97, 063525 (2018). arXiv: 1712.01760

19. Y. Akrami, R. Kallosh, A. Linde, V. Vardanyan, JCAP 1806, 041 (2018). arXiv: 1712.09693

20. A. Kehagias, A. Moradinezhad Dizgah, A. Riotto, Phys. Rev. D 89, 043527 (2014). arXiv:1312.1155

21. A. Masiero, M. Pietroni, F. Rosati, Phys. Rev. D 61, 023504 (1999). arXiv:hep-ph/9905346

22. M. Joy, V. Sahni, A.A. Starobinsky, Phys. Rev. D 77, 023514 (2008). arXiv:0711.1585

23. B.A. Bassett, S. Tsujikawa, D. Wands, Rev. Mod. Phys. 78, 537 (2006). arXiv:astro-ph/0507632

24. P.A.R. Ade et al., Astron. Astrophys. A 594, 13 (2016). arXiv: 1502.01589

25. D.J.H. Chung, E.W. Kolb, A. Riotto, Phys. Rev. D 59, 023501 (1998). arXiv:hep-ph/9802238

26. J. Haro, W. Yang, S. Pan, JCAP 01, 023 (2019). arXiv: 1811.07371

27. T.S. Bunch, J. Phys. A: Math. Gen. 13, 1297 (1980)

28. N.D. Birrell, C.P.W. Davies, J. Phys. A: Math. Gen. 13, 2109 (1980)

29. J. de Haro, J. Amorós, S. Pan, Phys. Rev. D 93, 084018 (2016). arXiv: 1601.08175

30. P.A.R. Ade et al., Astron. Astrophys. A 594, 13 (2016). arXiv: 1502.01589

31. J. Rubio, C. Wetterich, Phys. Rev. D 96, 063509 (2017). arXiv:1705.00552

32. J. Haro, J. Phys. A: Math. Theor. 44, 205401 (2011)

33. M. Wali Hossain, R. Myrzakulov, M. Sami, E.N. Saridakis, Int. J. Mod. Phys. D 24(05), 1530014 (2015). arXiv:1410.6100

34. G.F. Giudice, E.W. Kolb, A. Riotto, Phys. Rev. D 64, 023508 (2001). arXiv:hep-ph/0005123

35. G. Felder, L. Kofman, A. Linde, Phys. Rev. D 60, 103505 (1999). arXiv:hep-ph/9903350

36. J. Haro, Phys. Rev. D 99, 043510 (2019). arXiv:1807.07367

37. J. Haro, L. Aresté Saló, Phys. Rev. D 100, 043519 (2019). arXiv: 1906.02548

38. N. Bellomo, N. Bartolo, R. Jimenez, S. Matarrese, L. Verde, Measuring the energy scale of inflation with large scale structures. JCAP 11, 043 (2018). arXiv:1809.07113 